\title{
Doença de Addison de Etiologia Auto-Imune
}

\section{RESUMO}

A doença de Addison de etiologia auto-imune é uma endocrinopatia rara e potencialmente fatal, que pode ocorrer de forma isolada ou como parte das sindromes poliglandulares auto-imunes (SPA) dos tipo I e II. Auto-anticorpos anti-córtex adrenal são considerados marcadores imunológicos sensiveis do processo auto-imune destrutivo, podendo identificar individuos na fase pré-clínica da doença. A enzima 21-hidroxilase (citocromo P450c21) representa o principal antígeno adrenocortical, embora outros citocromos P450 ( $17 \alpha$-hidroxilase e colesterol desmolase) possam, também, desencadear a resposta auto-imune, principalmente na SPA do tipo I e na doença de Addison associada à falência ovariana precoce. O papel dos auto-anticorpos anti-P450c21 na patogênse da falência adrenal ainda não está bem estabelecido, assim como aquele dos anticorpos anti-receptor do ACTH. (Arq Bras Endocrinol Metab 1998;42/6:431-443).

Unitermos: Doença de Addison; Auto-imunidade; Auto-anticorpos; 21Hidroxilase; Síndromes poliglandulares.

\begin{abstract}
Autoimmune Addison's disease is a rare and potentially fatal endocrinopathy, that can occur either isolated or as part of the types I and $1 /$ polyglandular autoimmune syndromes (PAS). Adrenocortical autoantibodies are considered sensitive immunological markers of the destructive autoimmune process, and can identify individuals in the pre-clinical stage of the disease. The steroidogenic enzyme 21-hydroxylase ( $4450 \mathrm{C} 21)$ represents the major adrenal autoantigen, although other P450 cytochromes ( $17 \alpha$-hydroxylase and side chain cleavage) can also trigger an autoimmune response, mainly in the PAS type I and in Addison's disease with associated premature ovarian failure. The role of P45021 autoantibodies in the pathogenesis of the adrenal failure is not yet well established, and the same happens with the anti-ACTH receptor antibodies. (Arq Bras Endocrinol Metab 1998;42/6:431-443).
\end{abstract}

Keywords: Addison's disease: Autoimmunity; Autoantibodies; 21Hydroxylase; Polyglandular syndromes.

\section{DOENÇA DE ADDISON}

A insuficiênCIa adrenocortical. Primária crônica, originalmente descrita por Thomas Addison em 1855 (1), é uma endocrinopatia potencialmente fatal (2), com prevalência de 39 a 110 casos por milhão de habitantes (3-6). Resulta da destruição progressiva das células do córtex adrenal e se manifesta, predominantemente, como um quadro de insuficiência glico e mineralocorticóide $(7,8)$ [tabela 1]. Pode ser causada por infecçoes fúngicas (paracoccidiodomicose, histoplasmosc), bacterianas (tuberculose), virais (vírus da imunodeficiência adquirida, cito-

\author{
Regina C. Silva \\ Claudio E. Kater
}

Disciplina de Endocrinologia 0 Metabologia, Departamento de Medicina, Universidade Federal de São Paulo / Escola Paulista de Medicina, Săo Paulo, SP.
Recebido em $07 / 08 / 98$ Revisado em $19 / 09 / 98$ Aceito em $17 / 10 / 98$ 


\section{SÍNDROME POLIGLANDULAR AUTO-IMUNE DO TIPO II}

A DA como parte da SPA do tipo II se manifesta entre os 20 e 60 anos de idade e se associa, fundamentalmente, às doenças tiroidianas auto-imunes $(69 \%)$ e ao diabetes mellitus do tipo l $(50 \%)$ (13). A associação com o hipotiroidismo primário é a mais prevalente (21\% dos casos) e seu diagnóstico costuma ser feito concomitantemente ou após o desenvolvimento da DA (12). Diferentemente, o diabetes mellitus do tipo l costuma preceder o aparecimento da DA $(17,24)$. Outras patologias auto-imuncs não endócrinas também podem estar presentes nesta síndrome, como a doença celíaca, anemia perniciosa, vitiligo, alopécia c miastenia gravis $(13,17)$.

A prevalência da SPA do tipo II é de cerea de 15 a 45 casos por milhão, sendo mais freqüente em mulheres $(17,24)$. A susceptibilidade genética para o desenvolvimento desta síndrome é herdada de forma autossômica dominante, com penetrância variável $(24,25)$. Os genes responsáveis pela SPA do tipo II estão intimamente ligados a determinados alelos de risco nas classes I e II do sistema HLA, entre eles: B8 (26), $\mathrm{DQAl}^{*} 0501, \mathrm{DRB}{ }^{*} 0301$ e $\mathrm{DQBl}{ }^{*} 0201$, os quais são encontrados com maior freqüência nos pacientes que na população geral, mesmo na ausência de diabetes mellitus do tipo 1 (27-31).

O aminoácido existente na posição 57 do antígeno HLADQB (alanina, valina e serina, em substituição no ácido aspártico) também pode se correlacionar com maior susceptibilidade para a DA, por possibilitar às células apresentadoras de antígenos a apresentação de populações mais diversas de peptídeos às células $\mathrm{T}$, incluindo auto-antígenos (32).

Recentemente, o encontro de uma associação entre DA c um polimorfismo no gene ativo da 21 -hidroxilase (33) ou uma deleção de $30 \mathrm{~Kb}$ na classe III do complexo maior de histocompatibilidade, envolvendo o gene C4A do complemento e o pseudogene da 21 hidroxilase (31), fez supor que certas forma de 21 hidroxilase pudessem ser mais imunogênicas que outras. No entanto, esses marcadores específicos para DA na classe III do complexo HLA estavam sempre em desequilíbrio de ligação com os determinantes de risco de classe II (DRBl*030l, DQAl*050l e DQBl $\left.{ }^{*} 0201\right)$, tanto nos pacientes como nos controles (33).

Possivelmente, a susceptibilidade para DA, pelo menos em um subgrupo de pacientes definido por alelos de risco HLADQAl, também possa ser influenciada por genotipos do CTLA4 (gene que codifica o antígeno 4 dos linfócitos $\mathrm{T}$ citotóxicos ativados e que corresponde ao receptor da molécula co-estimulatória $\mathrm{B7}$, responsável pela regulação da interação dos linfócitos $\mathrm{T}$ com as células apresentadoras de antígeno, além de mediar a apoptose antígenoespecífica dos linfócitos T) (34).

\section{PATOLOGIA}

As glândulas adrenais de pacientes com DA de etiologia auto-imune são usualmente atróficas. Diferentemente do observado na DA secundária à tuberculose, o processo destrutivo auto-imune preserva a medula c altera a estrutura cortical. Em uma fase aguda, verifica-se a presença de extenso infiltrado de células mononucleares. Posteriormente, as poucas células adrenocorticais remanescentes vão sendo progressivamente substituídas por tecido fibroso $(35,36)$.

Dados de autópsia de indivíduos com mais de 60 anos de idade e sem DA revelaram a presença de infiltrado de células mononucleares em $63 \%$ dos casos, com predomínio de linfócitos $\mathrm{T} \mathrm{CD}^{+}{ }^{+}(24 \%$ deles ativados), o que pode refletir a ocorrência de auto-imunidade adrenal espontânea associada ao processo de envelhecimento ou a presença de adrenalite năo destrutiva (37).

As células adrenocorticais de portadores de DA de etiologia auto-imune de início recente (um dia a oito semanas) expressam de forma aberrante os antígenos de classe II do sistema HLA, o que as permite apresentarem antígenos ao sistema imune (38). Não está estabelecido, no entanto, se isso representa um cvento primário ou se é decorrente do estímulo inespecífico do interferon $-\gamma$, liberado pelo processo inflamatório (38). Da mesma forma, cabe ressaltar que cerca de $10 \%$ das células adrenocorticais normais da zona reticulada podem expressar de forma espontânea esses mesmos determinantes antigênicos (38).

A análise histopatológica fornece o diagnóstico de certeza da ctiologia auto-imune da DA. No entanto, a biópsia glandular é raramente realizada na prática clínica, por ser um procedimento invasivo. Os achados tomográficos ou de ressonância magnética de glândulas supra-renais normais ou com volume reduzido e sem calcificaçôes permitem diferenciar a DA de etiologia auto-imune daquela resultante de outras etiologias, principalmente as doenças granulomatosas (39-42).

\section{ASPECTOS DA IMUNIDADE CELULAR}

Embora ainda pouco estudado, é amplamente aceito que as células $T$ são fundamentais na patogênese da 
DA de etiologia auto-imune. À semelhança do que ocorre no diabetes mellitus do tipo 1 , acredita-se que a ativação de linfócitos $T$ "helper"(CD4+) autoreativos desempenhe um papel crítico nos estágios precoces da doença, levando à destruição celular através da ação de linfócitos T citotóxicos (CD8+). Em uma fase tardia, a liberação de citocinas pelos linfócitos $\mathrm{CD}^{+}$e consecuiente geração de radicais livres de oxigênio colaborariam para a manutenção do processo destrutivo inflamatório tecidual (43). Os fatores ambientais também podem modular a resposta imune através de uma ativação predominante de subpopulações de linfócitos CD4+ Thl (secretores de interleucina- 2 , fator de necrose tumoral- $\beta$ e interferon- $\gamma$ ) sobre as populaçoces Th2 (produtoras de interleucinas 4,5 c 10), o que tem sido implicado no maior estímulo da imunidade mediada por células em relação à humoral (44). Os linfócitos T supressores de pacientes com DA também são menos eficientes na inibição das células B c T (45).

Evidência de uma resposta antígeno-específica do linfócito $T$ pode ser demonstrada pelo teste de inibição da migração de leucócitos, que é um correlato in pitro da hipersensibilidade celular do tipo tardio. Quarenta e seis a $80 \%$ dos portadores de DA de etiologia autoimune apresentam macrófagos com menor capacidade de migração na presença de antígenos adrenais (devido à liberação dos inibidores de migração, interferong c interleucina 4 , pelos linfócitos $T$ estimulados), quando comparados a leucócitos de indivíduos normais ou com adrenalite secundária a tuberculose (46-49).

Estudos mais recentes, utilizando proténas adrenais purificadas e separadas de acordo com seu tamanho, mostraram uma resposta proliferativa dos linfócitos $T$ frente a uma fração contendo proteínas de peso molecular entre 18 e $24 \mathrm{KDa}$ em $60 \%$ dos casos de DA de etiologia auto-imune (50). Reforçando o conceito do cnvolvimento das células T na patogênese da DA de etiologia auto-imune, observou-se que o número relativo de linfócitos $\mathrm{T}$ ativados é duas a dez vezes maior em $100 \%$ dos pacientes com doença recém-diagnosticada (menos de um ano), quando comparado ao número cncontrado em controles saudáveis ou em portadores de DA de etiologia diferente da auto-imume, sugerindo processo auto-imune em atividade. Entretanto, pacientes com DA auto-imune com duração superior a cinco anos apresentam níveis normais de linfócitos $\mathrm{T}$ ativados circulantes $(51)$.

Em modelos animais, adrenalite auto-imune pode ser produzida pela imunização de camundongos com mistura de extrato de adrenal e lipopolissacíride de Klebsiella pneumoniae $\mathrm{O} 3$. Alćm disso, há aparecimen- to de anticorpos anti-córtex adrenal e o desenvolvimento de hipersensibilidade celular tardia. A transferência de esplenócitos dos camundongos imunizados com extrato de adrenal também ocasiona tanto o descnvolvimento de infiltrados no córtex adrenal como o aparecimento de anticorpos em receptores saudáveis, mas sem desencadear insuficiência adrenal. Isso indica que as lesões adrenais são induzidas através de mecanismo auto-imune e que a imunidade mediada por células desempenha um papel importante na produçăo dessas lesōes (52).

A caracterização, isolamento c clonagem das células do infiltrado inflamatório permitirĩo, no futuro, a identificação dos auto-epitopos das células T e uma melhor compreensão dos mecanismos celulares envolvidos na patogênese da DA de etiologia auto-imune.

\section{ASPECTOS DA IMUNIDADE HUMORAL}

\section{AUTO-ANTICORPOS ANTI-CÓRTEX ADRENAL E ANTI-CÉLULAS PRODUTORAS DE ESTERÓIDES}

A demonstração de auto-anticorpos anti-cóltex adrenal (ACA) circulantes, através de fixação de complemento e imunofluorescencia indireta (IFI), foi o primeiro passo importante para a definição da etiologia auto-imunc da DA $(53,54)$.

OS ACA sĩo imunoglobulinas da classe $(i$, convencionalmente detectadas através de IFI, em cortes criostáticos de adrenal humana ou bovina (55). Estão presentes no soro de $60 \%$ a $80 \%$ dos portadores de DA de etiologia idiopática (12,56-59), principalmente cm pacientes do sexo feminino e nos portadores de SPA $(60,61)$. Sula positividade costuma ser maior quanto menor a duração da doença. De acordo com Wuepper e cols (57), 89\% dos pacientes estudiados dentro de dois anos do diagnóstico têm ACA circulantes, comparados a $50 \%$ dos pacientes com mais de dois anos de duração da DA. Nerup (59) também encontrou ACA com frequiencia significantemente maior no soro de pacientes com menos de einco anos de duraçăo da doença $(83 \%$ vs $58 \%$ ). Da mesma forma, Falorni e cols (62) demonstraram a presença de ACA em 73\% dos pacientes com menos de 15 anos de duração da DA, comparado a $8 \%$ nos pacientes com diagnóstico há mais de 15 anos.

Além da IFI, outras técnicas podem ser utilizadas para a deteç̧ão dos ACA, entre clas: a ELISA (63), o radiomunoensaio (64) e a técnica da proténa-A imunoperoxidase (PAIP) (65). Esta última, além de apresentar sensibilidade e especificidade comparáveis à IFI, tem as vantagens de dispensar o uso do microscópio de imunofluorescência e de permitir o estoque das lâmi- 
nas por tempo prolongado (65). Tanto a técnica de IFI como a PAIP são semiquantitativas e sofiem interferência da qualidade do tecido adrenocortical utilizado, da habilidade do pesquisador e da presença no soro de lipemia e anticorpos anti-mitocondriais ou anti-ribossomais, fatores que podem tornar dificil a diferenciação entre um título fraco positivo c um negativo ("background") $(63,65)$.

Un subgrupo dos ACA apresenta reação cruzada com antígenos compartillados por outras células produtoras de esteróides presentes nos ovários (cćlulas da teca interna, hilares c luteinizadas), testículos (células de Leydig) e placenta (trofoblastos), sendo denominados anticorpos anti-células produtoras de esteróides (ACPE) (11,66-68). Os ACPE são raramente encontrados na ausência dos ACA (69). Estão presentes em $15 \%$ a $20 \%$ das paciontes com DA, sendo que $40 \%$ delas evoluem para falência ovariana em um período de 10 a 15 anos (70). São também detectados no soro de $71 \%$ a $86 \%$ dos portadorese de SPA do tipo I, correlacionando-se fortemente com a presença de falência ovariana precoce $(71,72)$.

Tanto os ACA como os ACPE são raramente encontrados na população geral $(59,69,73)$. Os ACA ocorrem $\mathrm{em} 10$ a $30 \%$ dos casos de hipoparatiroidismo idiopático e são um achado ocasional no soro de diabéticos do tipo le portadores de doenças auto-imuncs tiroidianas $(11,74)$. Quando detectados na ausência de DA clínica, esses anticorpos funcionam como marcadores de falência glandular $\mathrm{cm}$ potencial, principalmente se fixadores de complemento (75) e se detectados de forma persistente no plasma em títulos superiores a $1: 8(74,76)$.

A evoluçào para falência glandular passa por quatro estágios funcionais diferentes, sendo que a elevação da atividade plasmática da renina é a primcira anormalidade detectada, seguida de resposta subnormal do cortisol frente ao estímulo com ACTH exógeno, aumento dos níveis de ACTH e, finalmente, diminuição dos níveis plasmáticos de cortisol (77). No entanto, remissio espontânea pode ocorrer $\mathrm{cm} 30 \%$ dos portadores de docnças auto-imunes órgão-específicas e ACA em títulos inferiores a $1: 8$, especialmente nos estágios precoces da insuficiência adrenocortical subclínica. Da mesma forma, reversibilidade da disfunção adrenal também pode ser induzida por corticoterapia em dose immossupressora, apesar dos altos títulos de ACA (1:32 a 1:64), mesmo cm pacientes em cstágios mais avançados da insuficiência adrenocortical subclínica (74).

Os ACA estão usualmente ausentes no soro de portadores de DA de etiologia granulomatosa, embora haja relatos do encontro de ACA em 7\% a 18\% dos portado- res de DA secundária à tuberculose $(78,79)$, provavelmente devido à liberação de antígenos provocada pela resposta inflamatória ou à existência de dois mecanismos separados de lesĩo agindo simultaneamente.

\section{AUTO-ANTÍGENOS NA DOENÇA DE ADDISON (tabela 4)}

O antígeno-alvo dos ACA localiza-se no citoplasma das células das três camadas do córtex adrenal. A nível subcelular, a fração microssomal absorve de forma quantitativa o anticorpo do soro testado, embora também haja relatos de reação $\mathrm{com}$ a fração mitocondrial (80). Reatividade contra a superficie celular também foi demonstrada através de imunofluorescência de superfície em células adrenais intactas, havendo correlação entre anticorpos reativos contra o citoplasma e aqueles dirigidos contra a membrana citoplasmática (81). A ligação dos ACA a frações subcelulares ricas em citocromo c-rcdutase e 5'-nucleotidase (marcadores do microssoma c da membrana plasmática, respectivamente) também sugere que o antígeno possa estar representado na membrana plasmática $(82,83)$.

Tabela 4 - Auto-antigenos adrenais nas diferentes formas de doença de Addison.

\begin{tabular}{ll}
\hline Doença & Auto-antigeno adrenal \\
Doença de Addison isolada & 21-hidroxilase \\
SPA do tipo II & 21-hidroxilase \\
SPA do tipo I & 21-hidroxilase, 17 $\alpha$-hidroxilase \\
& e colesterol desmolase \\
Doença de Addison + & $17 \alpha$-hidroxilase, colesterol \\
Falência ovariana precoce & desmolase e 21-hidroxilase \\
\hline
\end{tabular}

Em 1988, Furmaniak e cols (84) demonstraram que $57 \%$ dos soros de portadores de DA de etiologia auto-imune c ACA positivo à IFI, imunoprecipitavam especificamente uma proteína adrenal microssomal de $55 \mathrm{KDa}$, sob condiçoes reduzidas, e uma de $58 \mathrm{KDa}$, sob condições não reduzidas. Posteriormente, a enzima $2 \mathrm{l}$-hidroxilase $(\mathrm{P} 450 \mathrm{c} 2 \mathrm{l}$ ) foi identificada como o principal antígeno na DA de etiologia auto-imune isolada e naquela associada à SPA do tipo II. Winquist $\mathrm{C}$ cols, em 1992 (85), observaram que o soro ACA positivo de 12 de 16 (75\%) portadores de DA de etiologia auto-imune isolada e de início na idade adulta reconheciam, no "immunoblotting" uma proteína da fração microssomal de adrenal bovina de $54 \mathrm{KDa}$, a qual migrava na cletroforese na mesma posição da P450c21. Da mesma forma, tanto os soros de portadores de DA de etiologia auto-imune isolada como os 
anticorpos de coelho anti-P450c2 1 recombinante reagiam especificamente com uma proteína adrenal de 55 KDa e com a P450c2 1 humana recombinante expressa em Saccharomyces cerevisae ou com a P450c2l nativa purificada (86).

A P450c2l ć uma enzima exclusiva do córtex adrenal, codificada por um gene localizado no cromossomo 6, na classe III do sistema HLA. Tem peso molecular de $55 \mathrm{KDa}$ e é constituída de 494 aminoácidos (87). Localiza-se no retículo endoplasmático e necessita de uma redutase dependente de NADPH para a sua atividade. Converte 17-hidroxiprogesterona em 1 1-deoxicortisol e progesterona em deoxicorticosterona (88). Mapeamento dos epitopos aos quais os auto-anticorpos anti-P450c21 se ligam foi possível através da expressão de fragmentos da P450c2 l, usando translação in vitro ou expressão bacteriana da enzima. Esses estudos indicaram que a reatividade dos anticorpos é restrita a sítios limitados da proteína. Em $90 \%$ dos casos, os anticorpos reconhecem a porção central da proteína (aminoácidos 165 a 379), sendo predominante o reconhecimento do epitopo localizado entre os resíduos 281 e 379 , onde se situa o sítio de ligação de esteróide (aminoácidos 342 a 358). Além disso, em metade dos casos, os anticorpos reagem com a porção carboxi-terminal (aminoácidos 380 a 494), a qual inclui o sítio de ligação do heme, conservado $\mathrm{cm}$ todas as enzimas citocromo $\mathrm{P} 450$ $(32,89,90)$. Até mesmo alteraçōes únicas de aminoácidos, no sítio de ligação esteróide ou heme, afetam marcantemente a ligação dos auto-anticorpos humanos, conforme demonstrado quando diferentes mutações que ocorrem normalmente na P450c2l (na hiperplasia adrenal congênita não clássica) foram testadas (Arg339'His: redução de 15\% e Pro453'Ser: redução superior a 40\%) (91). Tanaka e cols (92) obscrvaram redução de $50 \%$ na ligação dos auto-anticorpos anti-P450c2 1 após a produção de mutações na Cys 428 (para Ser, Arg e Phe); também, a mutação que ocorre naturalmente na Ile 172 (para Asn) causou redução de cerca 20\% na ligação dos anticorpos (92). De acordo com Wedlock e cols (89), os segmentos central e carboxi-terminal da P450c2l interagem para formar um epitopo conformacional (tridimensional), o qual é muito conservado e importante para a atividade enzimática. Há, dessa forma, uma pressão evolutiva para conservar seqüências de aminoácidos críticas para a atividade enzimática, apesar delas facilmente provocarem respostas auto-imunes indesejáveis.

Recentemente, Volpato e cols (93), estudando os epitopos reconhecidos por auto-anticorpos antiP450c21, não observaram diferenças no padrão de reatividade em pacientes com DA isolada ou com SPA dos tipos I e II e em indivíduos com auto-anticorpos e função adrenal normal ou DA subclínica, sugerindo que os soros de pacientes com diferentes formas de DA auto-imune também reconhecem os mesmos epitopos da região carboxi-terminal e central da P450c21. Isso difere do observado com os auto-anticorpos anti-GAD 65 , os quais são reativos contra diferentes epitopos em portadores de diabetes mellitus do tipo 1, quando comparados aos portadores de "stiffman syndrome" e SPA do tipo I (94).

A natureza dos ACPE também foi elucidada quando, após reação de uma biblioteca de cDNA de adrenal fetal com o soro de portadores de SPA do tipo I houve reconhecimento, no "immunoblotting", de uma proteína de peso molecular $55 \mathrm{KDa}$, a qual apresentava 99\% de homologia com a enzima $17 \alpha$-hidroxilase (P450c17) (95). Esta enzima está presente no retículo endoplasmático do córtex adrenal e gônadas (88). Quatro cpitopos distintos foram encontrados: ERI (aminoácidos 122-148), ER2 (aminoácidos 280-304), ER3 (aminoácidos 396-432) e ER4 (aminoácidos 466508) (96). Nos últimos 70 a 80 aminoácidos da proté́na localiza-se a região que inclui o sítio catalítico e a cisteína, essencial para o funcionamento de todos os citocromos P450 por se ligar ao grupo heme (95).

Posteriormente, verificou-se que o soro de portadores de SPA do tipo I também reagiam no "immunoblotting" com uma proténa de $53 \mathrm{KDa}$ da fração mitocondrial da adrenal bovina, a qual além de migrar na mesma posição da colesterol desmolase (side chain cleavage, P450scc) também era reconhecida por anticorpo de coelho anti-P450scc (97). A P450scc está presente no córtex adrenal, gônadas e placenta e requer uma adrenodoxina, adrenodoxina redutase e NADPH para sua atividade (88).

A homologia de apenas $16 \%$ na seqüência de aminoácidos entre a P450cl7 e a P450sce (97) e o fato de que o anticorpo anti-P450scc cora a zona glomerulosa na IFI, diferentemente do anticorpo antiP450c17 (a qual não é expressa na zona glomerulosa), falam contra a hipótese de que haveria uma reatividade cruzada entre a P450cl7 e a P450sce na SPA do tipo I e sugerem uma resposta imune heterogênea nesta síndrome (98). Dessa forma, os anticorpos antiP450cl7 e P450sce se constituem nos principais componentes dos ACPE (99).

$\mathrm{Na} S P A$ do tipo I, também foi descrita uma reposta anticorpal contra a P450c21 (99). De acordo com Uibo e cols (98), na SPA do tipo I (com DA), cerca de $80 \%$ dos soros reagem contra uma ou mais das três enzimas citocromo P450. 
A P450c21 e a P450cl7 são enzimas citocrômicas localizadas no retículo endoplasmático, com pesos moleculares semelhantes e cerca de $30 \%$ de homologia na seqüência de aminoácidos (principalmente nas regiōes catalíticas situadas na região carboxi-terminal) $(96,100)$. No entanto, em estudos de absorção, a ausência de reatividade cruzada entre os anticorpos para uma seqüência comum das duas enzimas suporta a idéia de uma reposta imune independente para cada citocromo P450 e sugere que cada enzima seja o alvo primário da imunidade humoral na SPA do tipo I (101).

Os diversos estudos realizados até o monento indicam que a P450c2l é o principal auto-antígeno na DA de etiologia auto-imune, independentemente de ser isolada ou associada às SPAs dos tipos I e II. Qualquer un dos três citocromos P450 pode desencadear o aparecimento da DA na SPA do tipo I, embora a $\mathrm{P} 450 \mathrm{c} 21$ pareça particularmente crítica para o desenvolvimento da DA isolada e aquela associada à SPA do tipo II (102).

Aproximadamente $20 \%$ dos soros de portadores de DA de etiologia idiopática não apresentam reatividade contra nenhuma das três enzimas citocromo P450 (102). Embora não tenha sido detectada reatividade contra outras enzimas, tais como a $11-\beta$-hidroxilase, a aromatase, a adrenodoxina e a $3 \beta$-hidroxiesteróide dehidrogenase, a hipótese de que existam auto-antígenos adicionais ainda não identificados não pode ser descartada (101, 103). Um antígeno de $51 \mathrm{KDa}$, localizado no retículo endoplasmático das células da granulosa e placenta, foi reconhecido pela maioria dos soros de portadores de DA e ACPE positivos (104). Uibo e cols (105) também observaram que o soro de portadores de SPA do tipo I identifica uma proteína adrenal de $43 \mathrm{KDa}$ ainda não identificada. Da mesma forma, síntese de auto-anticorpos localizada, sem evidência sorológica, e duração da doença devem ser levadas em consideração na tentativa de explicar esses casos onde nenhum anticorpo pode ser detectado (102).

\section{AVANÇOS DIAGNÓSTICOS}

A identificação dos auto-antígenos adrenocorticais possibilitou o desenvolvimento de técnicas mais específicas e sensíveis para o diagnóstico da DA de etiologia auto-imune, tais como os radioensaios. Nestes, o DNA complementar (cDNA) das enzimas esteroidogênicas humanas é utilizado em uma reação de transcrição/translação acopladas in vitro, em reticulócitos de coelho, onde se adiciona um aminoácido marcado com o isótopo radioativo ( ${ }^{3 \pi} \mathrm{S}$-metionina).
Posteriormente, as enzimas humanas recombinantes marcadas são imunoprecipitadas com o soros dos pacientes e as proténas ligadas aos anticorpos são scparadas das proteínas livres através da adição de proteína-A sefarose. A radioatividade é quantificada $\mathrm{em}$ um contador de líquido de cintilação $(99,106,107)$. Essa técnica é quantitativa, apresenta boa reprodutibilidade e especificidade e sensibilidade elevadas (devido ao uso do isótopo radioativo). A utilização de uma placa com 96 poços para a imunoprecipitação permite a análise simultânea de grande número de soros. A translação cucariótica possibilita a manutenção do epitopo conformacional e de possíveis modificações póstranslacionais, diferentemente do observado com enzimas expressas em bactérias. Os resultados são expressos como índices relativos, utilizando-se um padrão positivo e dois negativos, a fim de diminuir a variação inter-ensaio. As únicas limitações estão associadas com o uso de DNA e de material radioativo, o que implica num maior controle do ambiente, a fim de evitar contaminação.

Um novo radioensaio, baseado na utilização de P450c21 humana recombinante expressa $\mathrm{cm}$ Saccharomyces cerevisae e marcada com ${ }^{125} \mathrm{I}$ pelo método da cloramina $\mathrm{T}$, foi desenvolvido por Tanaka e cols (108), os quais obtiveram resultados comparáveis aos dos ensaios citados anteriormente.

Auto-anticorpos anti-P450c2l detectados por radioensaio apresentam boa correlação com aqueles detectados através de "immunoblotting" e com os ACA detectados por IFI. Diferentemente do "immunoblotting", no entanto, não existe o risco de desnaturar o antígeno e alterar o epitopo conformacional ao qual o anticorpo se liga. Não houve reação cruzada com outros anticorpos presentes no soro, tais como fator reumatóide, anticorpos anti-DNA de dupla fita, anti-receptor do TSH, anti-tiroglobulina, antiperoxidase e anti-GAD 65 (106).

Auto-anticorpos anti-P450c2l são raramente encontrados na população geral $(1,4 \%$ a $2,5 \%)$ $(107,108)$ e a sua prevalência em portadores de DA de etiologia auto-imune isolada varia de $64 \%$ a $89 \%$ (99,106-108). Os níveis de auto-anticorpos antiP450c2l costumam se correlacionar inversamente com a duração da falência adrenocortical de etiologia auto-imune: $100 \%$ dos pacientes com menos de 20 anos de duração da doença foram positivos, comparados a $67 \%$ dos pacientes com mais de 20 anos de duração da doença (107). Também, os auto-anticorpos anti-P450c2l são mais sensíveis que os $A C A$ detectados por IFI em pacientes com mais de 15 anos de duração da DA (62). 


\section{PAPEL DOS AUTO-ANTICORPOS ANTI-P450C21 NA PATOGÊNESE DA FALÊNCIA ADRENAL}

O papel dos auto-anticorpos anti-P450<2 I na patogênese da insuficiência adrenocortical primária não está bem estabelecido. É difícil imaginar anticorpos que possam reagir com antígenos intra-celulares em células intactas, mas se deve levar em consideração a possibilidade da expressão auto-antigênica na superfícic celular, principalmente como resultado de um estresse como o que ocorre durante infecções virais (32). Além do mais, há relatos da expressão antigênica na superfícic celular, o que talvez permita aos auto-anticorpos atuar na patogềnese da falência adrenocortical, através de citotoxicidade humoral mediada pelo complemento e citotoxicidade celular dependente de anticorpos, através das células killer $(81,82)$.

Estudos in vitro demonstraram que esses autoanticorpos são capazes de inibir a função enzimática (menor conversão de progesterona a deoxicorticosterona) (109). No entanto, não foi possível demonstrar a inibição da atividade cnzimática in vivo, pois pacientes com DA subclínica e auto-anticorpos positivos não apresentam nenhum acúmulo de 17 hidroxiprogesterona (indicador bioquímico da deficiência enzimática na hiperplasia adrenal congênita) (110,111). Portanto, parece que a destruição das células adrenocorticais mediada pelos linfócitos $T$ é central na patogênese da DA de etiologia autoimune e que a produção de auto-anticorpos antiP450c2 l é secundária à liberação de P450)2 l e peptídeos a ela relacionados. Dessa forma, os auto-anticorpos anti-P450c2l funcionam apenas como marcadores sorológicos do processo aluto-imune (epifenômeno), apesar de se ligaram a sítios importantes para a atividade enzimática. Laureti e cols (112), cntretanto, observaram que os níveis de auto-anticorpos anti-P450c21 se correlacionam com o grau de disfunção adrenal em indivíduos com insuficiência adrenal pré-clinica; isto sugere que a produção de altos níveis de anticorpos está associada com a ativação de uma fase destrutiva irreversível do processo auto-imune, c que os auto-anticorpos anti-P450c2 l são o resultado de um processo scletivo, oligoclonal e epitopo-específico capaz de gerar auto-anticorpos de alta afinidade pelo antígeno. Além disso, a ausência de reatividade do soro de portadores de SPA do tipo I contra a $11 \beta$-hidroxilase, a aromatase, a adrenodoxina e a $3 \beta$-hidroxiesteróide dehidrogenase (101), também fala contra o fato da imunidade humoral ser inespecífica.

\section{VALOR PROGNÓSTICO DOS AUTO-ANTICORPOS ANTI-P450c21, ANTI-P450c17 E ANTI-P450scC}

Devido à possibilidade de ocorrência de múltiplas cndocrinopatias auto-imunes no mesmo paciente, torna-se necessária a identificaçăo precoce de indivíduos de risco para o desenvolvimento de DA, através da dosagem de auto-anticorpos anti-P450c21.

Auto-anticorpos anti-P450c2l são detectados, através de radiocnsaio, em 0,6 a $2,3 \%$ dos portadores de diabetes mellitus do tipo 1 , sendo altamente sensíveis e específicos para o diagnóstico de DA $(62,113)$. Esses auto-anticorpos também sĩo detectados em $3 \%$ dos portadores de moléstia de Basedow Graves, apresentando um valor preditivo positivo para o diagnóstico de DA superior a $60 \%(62)$.

De acordo com Betterle e cols (114), 90\% das crianças portadoras de endocrinopatias auto-imunes e auto-anticorpos anti-P450c2l positivos evolnem para falência adienal após um período médio de latência de três anos e a progressão de pacientes do estágio zero (função adrenal normal) para DA clínica é rápida (14 meses). Diferentemente, $2 \mathbf{l} \%$ dos adultos portadores de endocrinpatias auto-imunes e auto-anticorpos antiP450c2l positivos evoluem para DA clínica após um perído médio de latência de trếs anos, mas a progressĩo do estágio zero para o estágio quatro (DA clínica) é mais lenta, ocorrendo em cerca de cinco anos (115). Em adultos, altos níveis de auto-anticorpos anti-P450c2l, função adrenal alterada na época da deteção do anticorpo e HLA-DR3 estão associados com maior progressão para DA clínica (115). Betterle e cols (115) recomendam a realizaçio anual do teste de estímulo rápido com ACTH $c$ a introdução de terapia substitutiva já no primciro estágio de disfunção adrenal (aumento da atividade plasmática da renina), a fim de previnir crise adrenal aguda nesses pacientes, principalmente durante um estresse ou infeção. A terapia substitutiva com glicocorticóides pode ser utilizada de forma profilática, para reduzir a função das células adrenocorticais c a expressio antigênica, de forma a lentificar ou até mesmo interromper o processo auto-imune, retardando o início clínico da doença. Além disso, terapia imunossupressora ou imunomoduladora também pode ser usada na tentativa de se bloquear o processo auto-imune, restituindo a função do órgão-alvo (32).

Os auto-anticorpos anti-P450cl7 e P450sec constituem-se $\mathrm{cm}$ marcadores sorológicos de falência gonadal em mulheres, mas não en homens, sendo o testículo mais resistente à destruição auto-imune, possivelmente devido à proteção oferecida pela barreira 
hêmato-testicular, bem como devido a fatores genéticos c hormonais $(30,104)$.

\section{ANTICORPOS ANTI-RECEPTOR DO ACTH}

Existem anticorpos contra receptores hormonais ou determinantes antigênicos intimamente relacionados com esses receptores, os quais podem bloqucar a ação hormonal e corroborar na patogênese da DA de etiologia auto-imune (83).

Anticorpos anti-receptor do ACTH podem bloquear a síntese de DNA e/ou o cstímulo da produçióo de cortisol pelo ACTH, antes da geração do AMP cíclico, sem um cfeito dircto citopático (116). Podem, também, agir conjuntamente com os ACA c a inunidade celular, afetando o trofismo glandular (117). Especificamente, IgGs que bloqueiam a ação do ACTH estimuladora do crescimento adrenal foram detectadas em $80 \%$ dos portadores de DA de etiologia auto-imune, enquanto que IgGs bloqucadoras dos efeitos esteroidogênicos do ACTH foram detectadas em $74 \%$ dos casos $(83,117)$. Esses dados, no entanto, não foram confirmados em outros estudos (118).

\section{CONCLUSŌES}

Na maioria dos casos, a DA resulta da destruição autoimune das células do córtex adrenal. Os ACA correspondem a marcadores sensíveis e específicos desse processo auto-imune destrutivo, sendo úteis para a identificação de indivíduos de risco para o desenvolvimento de falência adrenal. A enzima P450c2l foi recentemente identificada como o principal autoantígeno na DA de ctiologia auto-imune, cmbora outros citocromos P450 (P450cl7 c P450sce) tam bém possam desencadear o processo auto-imune, principalmente na SPA do tipo I e na DA associada à falência ovariana precoce. Apesar desses auto-anticorpos se ligarem a sítios importantes para a atividade enzimática, seu papel na patogênese da falência adrenal ainda não cstá bem estabelecido e a maioria dos estudos aponta para uma atuação predominante dos linfócitos T citotóxicos, causando destruição das células adrenocorticais c subseqüente falência glandular.

A descoberta de novos determinantes genéticos, dentro ou fora do sistema HLA, os quais predispóen à perda da tolerância imunológica c ao aparecimento da auto-imunidade anti-adrenocortical ainda se faz necessária. A deteç̧ão precoce de auto-anticorpos anti -P450c21, anti-P450c17 c anti-P450sce, somada à melhor comprecnsão dos mecanismos etiopatogênicos, certamente permitirão, num futuro próximo, a introdução de terapias isohormonais ou imunoterapias preventivas especificas.

\section{REFERÊNCIAS}

1. Addison T. On the constitutional and local effects of disease of the suprarenal capsules. Med Classics 1937; 2: 244-293.

2. Ringstad J, Rodge S, Loland $W$, Rode L. Case report. Rapidly fatal Addison's disease: three case reports. J Intern Med 1991; 230: 465-467.

3. Stuart-Mason A, Meadde TW, Lee JAH, Morris JN. Epidemiological and clinical picture of Addison's disease. Lancet 1968; 2: 744-747.

4. Nerup J. Addison's disease - a review of some clinical, pathological and immunological features. Dan Med Bull 1974; 21: 201-217.

5. Willis AC, Vince FP. The prevalence of Addison's disease in Coventry, UK. Postgrad Med J 1997: 73: 286-288.

6. Kong MF, Jeffcoat W. Eighty-six cases of Addison's disease. Clin Endocrinol 1994; 41: 757-761.

7. Oelkers W. Adrenal insufficiency. N Engl J Med 1996; 335: $1206-1212$.

8. Kater CE, Faiçal S, Zanella MT. Como reconhecer e tratar a insuficiência adrenocortical. J Bras Med 1993; 64: 168-170.

9. Colombo AL, Faiçal S, Kater CE. Systematic evaluation of the adrenocortical function in patients with paracoccidiodomycosis. Mycopathologia 1994; 127: 89-93.

10. Lewi DS, Kater CE. Insuficienncia adrenocortical em pacientes com sindrome de imunodeficiência adquirida (AIDS). Rev Assoc Med Brasil 1988; 34: 213-218.

11. Irvine WJ, Barnes EW. Addison's disease, ovarian failure and hypoparathyroidism. Clin Endocrinol Metab 1975; 4: $379-434$.

12. Zelissen PMJ, Bast EJEG, Croughs RJM. Associated autoimmunity in Addison's diasese. J Autoimmunity $1995 ; 8: 121-130$.

13. Neufeld M, Maclaren NK, Blizzard RM. Two types of autoimmune Addison's disease associated with different polyglandular autoimmune (PGA) syndromes. Medicine 1981; 60: 355-362.

14. Neufeld M. Maclaren N, Blizzard R. Autoimmune polyglandular syndromes. Pediatr Annals 1980: 9: 154-163.

15. Ahonen P. Myllärniemi S, Sipilä I, Perheentupa J. Clinical variation of autoimmune polvendocrinopathy - candidiasis - ectodermal dystrophy (APECED) in a series of 68 patients. N Engl J Med 1990; 322: 1829-1836.

16. Betterle C, Greggio NA, Volpato M. Autoimmune Polyglandular Syndrome type I. J Clin Endocrinol Metab 1998; 83: 1049-1055.

17. Betterle C, Volpato M. Adrenal and ovarian autoimmu nity. Eur J Endocrinol 1998; 138: 16-25.

18. Hayashida CY, Toledo SPA, Barros MT, EzabellaMCL, Laudanna AA. Síndrome poliglandular autoimune do tipo 1 com hipoparatiroidismo, candidiase mucocutânea e malabsorção intestinal. Rev Hosp Clin Fac Med S Paulo 1990; 45: 24-28. 
19. Tuomi T, Björsees P, Falorni A, Partanen J, Perheentupa J, Lernmark $A$, et al . Antibodies to glutamic acid decarboxylase and insulin-dependent diabetes in patients with autoimmune polyendocrine syndrome type I. J Clin Endocrinol Metab 1996; 81: 1488-1494.

20. Ahonen P. Koskimies S, Lokki ML, Tillikainen A. Perheentupa J. The expression of autoimmune polyg landular disease type I appears associated with several HLA-A antigens but not with HLA-DR. J Clin Endocrinol Metab 1988; 66: 1152-1157.

21. Arulanantham K, Dwyer JM, Genel M. Evidence for defective immunoregulation in the syndrome of familial candidiasis endocrinopathy. N Engl J Med 1979: 300: 164- 168.

22. Aaitonen J, Björses P. Sandkuijl L, Perheentupa J. Peltonen $L$. An autosomal locus causing autoimmune disease: autoimmune polyglandular disease type । assigned to chromosome 21. Nat Genet 1994; 8: 83- 87.

23. Nagamine K, Peterson P. Scott HS, Kudoh J, Minoshima $S$. Heino $M$, et al. Positional cloning of the APECED gene. Nat Genet 1997; 17: 393-398.

24. Betterle C, Volpato M, Greggio NA. Presotto F. Type 2 polyglandular autoimmune disease (Schmidt's syndrome). J Pediatr Endocrinol Metab 1996; 9: 113-123.

25. Meyerson J, Lechuga-Gomes EE, Bigazzi PE, Walfish PG. Polyglandular autoimmune syndrome: current concepts. Can Med Assoc J 1988; 138: 604-612.

26. Eisenbarth $G$, Wilson P, Ward F, Lebovitz HE. HLA type and occurence of disease in familial polvglandular failure. N Engl J Med 1978; 298: 92-94.

27. Maclaren NK, Riley WJ. Inherited susceptibility to autoimmunhe Addison's disease is linked of human leucocyte antigens-DR3 and/or DR4, except when associated with type I autoimmune polyglandular syndrome. J Clin Endocrinol Metab 1986; 62: 455- 459

28. Weetman AP, Zhang L. Tandon N. Edwards OM. HLA associations with autoimmune Addison's disease. Tissue Antigens 1991: 38: 31-33.

29. Latinne D, Vanderput $Y$, De Bruyere M, Bottazzo GF Sokal $G$, Crabbe J. Addison's disease: immunological aspects. Tissue Antigens 1987; 30: 23-24.

30. Weetman AP. Autoimmunity to steroid-producing cells and familial polyendocrine autoimmunity. Baillière's Clin Endocrinol Metab 1995; 9: 157-174.

31. Partanen J, Peterson P, Westman P, Aranko S, Krohn K. Major histocompatibility complex class II and III in Addison's disease. MHC alleles do not predict autoantibody specificity and 21-hydroxylase gene polymorphism has no independet role in disease susceptibility. Hum Immunol 1994; 41: 135-140.

32. Winqvist $O$, Söderbergh $A$, Kämpe $O$. The autoimmune basis of adrenocortical destruction in Addison's disease. Mol Med Today 1996: 2: 282-289.

33. Peterson P, Partanen J, Aavik E, Salmi H, Pelkonen R, Krohn KJE. Steroid 21-hydroxylase gene polymorphism in Addison's disease patients. Tissue Antigens 1995; 46: 63-67.

34. Donner H. Braun J, Seidl C. Rau H, Finke R, Ventz M, et al. Codon 17 polymorphism of the cytotoxic T Iymphocyte antigen 4 gene in Hashimoto's thyroiditis and Addison's disese. J Clin Endocrinol Metab 1997; 82: 4130-4132.

35. Brenner $O$. Addison's disease with atrophy of the cortex of suprarenals. Q J Med 1928: 22: 121-144.
36. Petri M. Nerup J. Addison's adrenalitis. Studies on diffuse lymphocytic adrenalitis (idiopathic Addison's disease) and focal lymphocytic infiltration in a control material. Acta Pathol Microbiol Scand 1971; 79: 381-388.

37. Hayashi Y. Hioshi T, Takemura T, Kurashima C, Hirokawa $K$. Focal lymphocytic infiltration in the adrenal cortex in the elderly: immunohistological analysis of infiltrating lymphocytes. Clin Exp Immunol 1989: 77: 101-105.

38. Jackson R, McNicol AM, Farquharson M, Foulis AK. Class II MHC expression in normal adrenal cortex and cortical cells in autoimmune Addison's disease. J Pathol 1988; 155: 113-120.

39. Moulton JS, Moulton JS. CT of the adrenal glands. Semin Roentgenol 1988; 23: 288-303.

40. Doppman JL, Gill JR, Nienhuis AW, Earl JM, Long JA. CT findings in Addison's disease. J Comput Assist Tomography 1982; 6: 757-761.

41. De la Veja MS, Artero CV, Mías EM, Real JMF, Ramón JS. Importancia de la tomografia axial computadorizada en el diagnóstico etiológico de la enfermedad de Addison. Rev Clín Esp 1992: 190: 177-180.

42. Vita JA, Silverberg SJ, Goland RS, Austin JHM, Knowlton Al. Clinical clues to the cause of Addison's disease. Am $J$ Med 1985; 78: 461-466.

43. Calcinaro F, Lafferty KF, Shehadeh NN. Inflammatory mediators and development of autoimmune diabetes. In: Eisenbarth $G$, Lafferty KJ, editors. Type I diabetes. Cary: Oxford University Press, 1996: 91-118.

44. Papanicolou DA. Cytokines and adrenal insufficiency. Curr Opin Endocrinol Diabetes 1997; 4: 194-198.

45. Fairchild RS, Schimke RN, Abdou NI. Immunoregulation abnormalities in familial Addison's disease. J Clin Endocrinol Melab 1980; 51: 1074-1077.

46. Bendixen $G$, Soborg M. A leucocyte migration technique for in vitro detection of cellular (delayed type) hypersensitivity in man. Dan Med Bull 1969; 16: 1-6.

47. Weiser WY, Temple PA, Witek-Giannotti JS, Remold HG. Clark SC, David JR. Molecular cloning of a CDNA enconding a human macrophage migration inhibitory factor. Proc Natl Acad Sci USA 1989: 86: 7522-7526.

48. Nerup J. Andersen V. Bendixen $G$. Anti-adrenal cellular hypersensitivity in Addison's disease. Clin Exp Immunol 1969; 4: 355-363.

49. Nerup J, Bendixen $G$. Anti-adrenal cellular hypersensitivity in Addison's disease. Il. Correlation with clinical and serological findings. Clin Exp Immunol 1969; 5: 341-353.

50. Freeman $M$, Weetman $A P$. T and $B$ cell reactivity to adrenal antigens in autoimmune Addison's disease. Clin Exp Immunol 1992; 88: 275-279.

51. Rabinowe SL, Jackson RA, Dluhy RG, Williams GH. Ia-positive $T$ lymphocytes in recently diagniosed idiopathic Addison's disease. Am J Med 1984; 77: 597-601.

52. Fujii $Y$, Kato $N$, Kito J, Asai J, Yokochi T. Experimental autoimmune adrenalitis: a murine model for Addison's disease. Autoimmunity 1992; 12: 47-52.

53. Anderson JR, Goudie RB, Gray KG, Timbury GC. Auto-antibodies in Addison's disease. Lancet 1957; 1: 1123-1124.

54. Blizzard RM, Kyle MA, Chandler RW, Hung W. Adrenai antibodies in Addison's disease. Lancet 1962; 2:901-903. 
55. Irvine WJ. Stewart AG, Scarth L. A clinical and immunological sutdy of adrenocortical insufficiency (Addison's disease). Clin Exp Immunol 1967; 2: 31-69.

56. Irvine WJ, Barnes EW. Adrenocortical insufficiency. Clin Endocrinol Metab 1972; 1: 549-594.

57. Wuepper KD, Wegienka LC, Fudenberg HH. Immunological aspects of adrenocortical insufficiency. Am J Med 1969; 46: 206-216.

58. Maisey MN, Lessof MH. Addison's disease: a clinical study. Guy's Hosp Rep 1969; 1 18: 363-372.

59. Nerup J. Addison's disease - serological sutdies. Acta Endocrinol 1974c: 76: 142-158.

60. Blizzard RM, Chee D. Davis W. The incidence of adrenal and other antibodies in the sera of patients with idio pathic adrenal insufficiency (Addison's disease). Clin Exp Immunol 1967; 2: 19-30.

61. Papadopoulos KI, Hallengren B. Polvglandular autoimmune syndrome type $\|$ in patients with idioparhic Addison's disease. Acta Endocrinol 1990; 122: 472-478.

62. Falorni A, Laureti S, Nikoshkov A, Picchio ML, Hallengren B. Vandewalles $\mathrm{CL}$, et al. 21-hydroxylase autoantibodies in adult patients with endocrine autoimmune diseases are highly specific for Addison's disease. Clin Exp Immunol 1997; 107: 341-346.

63. Stechemesser E, Scherbaum WA, Grossmann T, Berg PA. An ELISA method for the detection of autoantibodies to adrenal cortex. J Immunol Methods 1985; 80: 67-76.

64. Kosowicz J, Gryczynska M. Bottazzo GF. A radioimmunoassay for the detection of adrenal autoantibodies. Clin Exp Immunol 1986; 63: 671-679.

65. Silva RS, Faiçal S, Laureti S, Falorni A, Dib SA, Kater CE. Detection of adrenocortical autoantibodies in Addison's disease with a peroxidase-labelled protein A technique. Braz J Med Biol Res 1998; 31: 1141-1148.

66. Anderson JR, Goudie RB, Gray K, Stuart-Smith DA. Immunological features of idiopathic Addison's disease: an antibody to cells producing steroid hormones. Clin Exp Immunol 1968; 3: 107-117.

67. Irvine WJ, Chan MMW, Scarth L. The further characterization of autoantibodies reactive with extra-adrenal steroid-producing cells in patients with adrenal disordes. Clin Exp Immunol 1969; 4: 489-503.

68. Irvine WJ, Chan MMW, Scarth L. Immunological aspects of premature ovarian failure associated with Addison's disease. Lancet 1968; ii: 883-887.

69. Elder M. Maclaren N, Riley W. Gonadal autoantibodies in patients with hypogonadism and/or Addison's disease. J Clin Endocrinol Metab 1981: 52: 1137-1142.

70. Betterle C, Rossi A, Dalla Pria S, Artifoni A, Pedini B, Gavasso $S$, et al. Premature ovarian failure: autoimmunity and natural history. Clin Endocrinol (Oxf) 1993; 39: 35-43.

71. Ahonen P. Miettinen A, Perheentupa J. Adrenal and steroidal cell antibodies in patients with autoimmune polyglandular disease type $\mathrm{I}$ and risk of adrenocortical and ovarian failure. J Clin Endocrinol Metab 1987; 64: 494-500.

72. Hoek A, Schoemaker J, Drexhage HA. Premature ovarian failure and ovarian autoimmunity. End Rev 1997; 18: 107-134.
73. Sotsiou F, Bottazzo GF, Doniach D. Immunofluorescence studies on autoantibodies to steroid-producing cells and to germline cells in endocrine disease and infertility. Clin Exp Immunol 1980; 39: 97-111.

74. De Bellis A, Bizzarro A, Rossi R, Amoresano Paglionico $V$. Criscuolo T, Lombardi $G$, Bellastella A. Remission of subclinical adrenocortical failure in subjects with adrenal autoantibodies. J Clin Endocrinol Metab 1993; 76: 1002-1007

75. Betterle C, Zanchetta R, Trevisan A, Zanette F, Pedini B, Mantero F. Complement-fixing adrenal autoantibodies as a marker for predicting onset of idiopathic Addison's disease, Lancet 1983; 1983; i: 1238-1241.

76. Scherbaum WA, Berg PA. Development of adrenocortical failure in non-addisonian patients with antibodies to adrenal cortex. Clin Endocrinol 1982; 16: 345-352.

77. Betterle C, Scalici C, Presotto F, Pedini B, Moro L, Rigon F, ef al. The natural history of adrenal function in autoimmune patients with adrenal autoantibodies. Endocrinol 1988; 117: 467-475.

78. Pousset $G$, Monier JC, Thivolet J. Anticorps antisurrénaliens et maladie d'Addison. Application de la technique d'immunofluorescence dans 100 cas d'insuffisance surrénale. Ann Endocrinol 1970; 31: 995-1002

79. Goudie RB, Anderson JR, Gray KK, Whyte G. Autoantibodies in Addison's disease. Lancel 1966: i: 1173-1176.

80. Goudie RB, MCDonald E, Anderson JR, Gray K Immunological features of idiopathic Addison's disease: characterization of the adrenocortical antigens. Clin Exp Immunol 1968; 3: 119-131.

81. Khoury EL, Hammond L, Bottazzo GF, Doniach D. Surface-reactive antibodies to human adrenal cells in Addison's disease. Clin Exp Immunol 1981: 45: 48-55.

82. Bright GM. Singh I. Adrenal autoantibodies bind to adrenal subcellular fractions enriched in cytochrome-c reductase and 5'-nucleotidase. J Clin Endocrinol Metab 1990; 70: 95-99.

83. Wulffraat NM, Drexhage HA, Bottazzo GF. Autoimmune aspects of Addison's disease. In: James VHT, editor. The Adrenal Gland. $2^{\text {nd }}$ ed. New York: Raven Press, 1992: 263-288.

84. Furmaniak J, Talbot D, Reinwein D. Benker $G$, Creach FM, Rees Smith B. Immunoprecipitation of human adrenat microsomal antigen. FEBS Let 1988; 231:25-28.

85. Winqvist $O$, Andres Karlsson F, Kämpe 0.21 -hydroxylase a major autoantigen in idiopathic Addison's disease. Lancet 1992; 339: 1559-1562

86. Baumann-Antczak A, Wedlock $N$, Bednarek J, Kiso $Y$ Krishnan $H$, Fowler $S$, et al. Autoimmune Addison's disease and 21-hydroxylase. Lancet 1992; 340: 429-430.

87. White PC, New MI, DuPont B. Structure of human steroid 21-hydroxylase genes. Proc Natl Acad Sci USA 1986: 83:5111-5115.

88. Simpson ER, Waterman MR. Regulation of expression of adrenocortical enzymes. In: James VHT, editor. The Adrenal Gland. $2^{\text {nd }}$ ed. New York: Raven Press, 1992: 191-207.

89. Wedlock N, Asawa T, Baumann-Antczak A, Rees Smith B, Furmaniak J. Autoimmune Addison's disease. Analysis of autoantibody binding sites on human steroid 21 -hydroxylase. FEBS Let 1993; 332: 123-126. 
90. Song $Y H$, Connor EL, Muir A, She JX, Zorovich B, Derovanesian D, et al. Autoantibody epitope mapping of the 21-hydroxylase antigen in autoimmune Addison's disease. J Clin Endocrinol Metab 1994; 78: 1108-1112.

91. Asawa T, Wedlock N, Baumann-Antczak A, Rees Smith $B$, Furmaniak J. Naturaliy occurring mutations in human steroid 21-hydroxylase influence adrenal autoantibody binding. J Clin Endocrinol Metab 1994; 79: 372-376.

92. Tanaka H. Asawa T, Powell M, Chen S, Rees Smith B, Furmaniak J. Autoantibody binding to steroid 21-hydroxylase - effect of five mutations. Autoimmunity 1997; 26 : 253-259.

93. Volpato M, Prentice L, Chen S, Betterle C, Rees Smith B, Furmaniak J. A sutdy of the epitopes on steroid 21hydroxylase recognized by autoantibodies in patients with or without Addison's disease. Clin Exp Immunol 1998; $111: 422-428$.

94. Björk E, Velloso LA, Kämpe O, Karlsson FA, GAD autoantibodies in insulin-dependent diabetes mellitus, stiffman syndrome and autoimmune polyendocrine syndrome type I recognize different epitopes. Diabetes 1994; 43: 161-165.

95. Krohn K, Uibo R, Aavik E, Peterson P, Savilahti K. Identification by molecular cloning of an autoantigen associated with Addison's disease as steroid $17 \mathrm{a}$ hydroxylase. Lancet 1992; 339: 770-773.

96. Peterson P, Krohn KJE. Mapping of B cell epitopes on steroid 17 a-hydroxylase, an autoantigen in autoimmune polygiandular syndrome type I. Clin Exp Immunol 1994; 98: 104-109.

97. Winqvist 0 , Gustafsson J, Rorsman F, Anders Karlsson F, Kämpe $O$. Two different cytochrome P450 enzymes are the adrenal antigens in autoimmune polyendocrine syndrome type I and Addison's disease. J Clin Invest 1993: 92: 2377-2385

98. Uibo R, Aavik E, Peterson P, Perheentupa J, Aranko S, Pelkonen R, Krohn KJE, Autoantibodies to cytochrome P450 enzymes P450scc, P450c17 and P450c21 in autoimmune polyglandular disease types I and II and in isolated Addison's disease. J Clin Endocrinol Metab 1994: 78: 323-328.

99. Chen S. Sawicka J, Betterle C, Powell M. Prentice L, Volpato M. Rees Smith B. Furmaniak J. Autoantibodies to steroidogenic enzymes in autoimmune polyglandular syndrome. Addison's disease, and premature ovarian failure. J Clin Endocrinol Metab 1996; 81: 1871-1876.

100. Picado-Leonard J, Miller W. Cloning and sequence of the human gene for P450cl7 (steroid 17 alpha-hydroxylase/17,20 lyase): similarify with the gene for P450c21. DNA 1987; 6: 439-448

101. Peterson P. Uibo R, Peränen J, Krohn K. immunoprecipitation of steroidogenic enzyme autoantigens with autoimmune polyglandular syndrome type I (APS I) sera; further evidence for independent humoral immunity to P450c17 and P450c21. Clin Exp Immunol 1997: 107: 335-340.

102. Weetman AP. Autoantigens in Addison's disease and associated syndromes. Clin Exp Immunol 1997; 107: 227-229.

103. Arif S, Vallian S, Farzaneh F, Zanone MM, James SL, Pietropaolo $M$, et al. Identification of 3b-hydroxysteroid dehydrogenase as a novel target of steroid cell autoantibodies: association of autoantibodies with endocrine autoimmune disease. J Clin Endocrinol Metab 1996; 81: 4439-4445.

104. Winqvist O, Gebre-Medhin G. Gustafsson J, Rítzen EM, Lundkvist $O ̈$. Anders Karlsson $F$, Kämpe $O$. Identification of the main gonadal autoantigens in patients with adrenal insufficiency and associated ovarian failure. J Clin Endocrinol Metab 1995; 80: 1717-1723.

105. Uibo R, Perheentupa J, Ovod V, Krohn KJE. Characterization of adrenal autoantigens recognized by sera from patients with autoimmune polyglandular syndrome (APS) type 1. J Autoimmunity 1994; 7: 399-411.

106. Colls J, Betterle C, Volpato M. Prentice L, Rees Smith B, Furmaniak J. immunoprecipitation assay for autoantibodies to steroid 21-hydroxylase in autoimmune adrenal diseases. Clin Chem 1995; 41: 375-380.

107. Falorni A, Nikoshkov A, Laureti S, Grenbäck E, Hulting AL, Casucci $G$, st al. High diagnostic accuracy for idioparhic Addison's disease with a sensitive radiobinding assay for autoantibodies against recombinant human 21-hydroxylase. J Clin Endocrinol Metab 1995; 80: $2752-2755$

108. Tanaka H, Perez MS, Powell M, Sanders JF, Sawicka J, Chen S, et al. Steroid 21-hydroxylase autoantibodies: measurements with a new immunoprecipitation assay. $\mathrm{J}$ Clin Endocrinol Metab 1997; 82: 1440-7446.

109. Furmaniak J, Kominami S, Asawa T, Wedlock N, Colls J, Rees Smith B. Autoimmune Addison's disease Evidence for a role of steroid 21-hydroxylase autoantibodies in adrenal insufficiency. J Clin Endocrinol Metab 1994: 79: 1517-1521.

110. Boscaro M, Betterle C, Sonino N, Volpato M, Paoletta A. Fallo F. Early adrenal hypofunction in patients with organ-specific autoantibodies and no clinical adrenal insufficiency. J Clin Endocrinol Metab 1994; 79: 452-455.

111. Boscaro M. Betterle C. Volpato M. Fallo F, Furmaniak J. Rees Smith B, et al. Hormonal responses during various phases of autoimmune adrenal failure: no evidence for 21 -hydroxylase enzyme activity inhibition in vivo. J Clin Endocrinol Metab 1996; 81: 2801-2804

112. Laureti S, De Bellis A, Muccitelli VI, Calcinaro F, Bizzarro A. Rossi R, et al . Levels of adrenocortical autoantibodies correlate with the degree of adrenal dysfunction in subjects with pre-clinical Addison's disease. J Clin Endocrinol Metab (in press).

113. Peterson P, Salmi H, Hyöty H, Miettinen A, llonen J. Reijonen $\mathrm{H}$, ef al. Steroid 2 I-hydroxylase autoantibodies in insulin-dependent diabetes mellitus. Clin Immunol Immunopathol 1997: 82: 37-42.

114. Betterle C, Volpato M, Rees Smith B, Furmaniak J, Chen $S$, Zanchetta R, et al. II. Adrenal cortex and steroid 21hydroxylase autoantibodies in children with organ-specific autoimmune diseases: markers of high progression to clinical Addison's disease. J Clin Endocrinol Metab 1997: 82: 939-942.

115. Betterle C, Volpato M, Rees Smith B. Furmaniak J, Chen S, Greggio NA, et al. I. Adrenal cortex and steroid 12hydroxylase autoantibodies in adult patients with organ-specific autoimmune diseases: markers of low progression to clinical Addison's disease. I Clin Endocrinol Metab 1997; 82: 932-938 
116. Kendall-Taylor P, Lambert A, Mitchell R, Robertson WR. Antibody that blocks stimulation of cortisol secretion by adrenocorticotrophic hormone in Addison's disease. $\mathrm{Br}$ Med J 1988: 1489-1491.

117. Wulffraat NM, Drexhage HA, Bottazzo GF, Wiersinga WM, Jeucken P. Van der Gaag R. Immunoglobulins of patients with idiopathic Addison's disease block the in vitro action of adrenocorticotropin. J Clin Endocrinol Metab 1989: 69: 231-2238.

118. Wardle CA, Weetamn AP, Mitchell R, Peers N, Robertson WR. Adrenocorticotropic hormone receptorblocking immunoglobulins in serum from patients with
Addison's disease: a reexamination. J Clin Endocrinol Melab 1993; 77: 75-753

\section{Endereço para correspondência:}

Regina do Carmo Silva

Disciplina de Endocrinologia

Universidade Federal de São Paulo.

Rua Botucatu, 740 - $2^{2}$ andar

04034-970 Säo Paulo, SP

FAX: (011) 570-6636

e-mail: rcarmo@mandic.com.br. 